Reprod. Nutr. Dévelop., 1982, 22 (1 B), 179-191.

\title{
Biochimie des lipides cérébraux (plus particulièrement des acides gras). Synthèse in situ et origine exogène au cours du développement. Quelques aspects de l'influence de la nutrition
}

\section{J. M. BOURRE}

Laboratoire de Neurochimie, $U$ 134, INSERM,

Hôpital de la Salpêtrière, 47, boulevard de l'Hopital, 75651 Paris Cedex 131.

Summary. Biochemistry of brain lipids (especially fatty acids): in situ biosynthesis and exogenous origin during development and some aspects of nutritional influence.

In contrast with other tissues, the nervous system is very rich in lipids, most of which are found in membranes. Fatty acids thus play a role in membrane structure and function : sphingolipids are essentially found in myelin and present original fatty acid patterns. Saturated and monounsaturated fatty acids are synthesized in microsomes by 3 different systems which differ at the level of the condensing enzyme. The activities of these systems are directly related to myelination. Mitochondria are also able to synthesize fatty acids but the pathways are totally different and unrelated to myelination.

Moreover, the brain does not elaborate all its membrane fatty acids whose exogenous origin is demonstrated by injecting labelled non-essential fatty acids. The relationship between blood and brain vary during brain development; the uptake of fatty acids is quantitatively very important during glial cell multiplication and myelination.

Nutrition alters the fatty acid composition of brain membranes; the fatty acids are largely altered in an opposite way in the neurons and oligodendrocytes of hypotrophic animals.

\section{Les lipides cérébraux}

Les lipides furent longtemps définis par une de leurs propriétés physiques : leur solubilité dans les solvants organiques. Actuellement on préfère les caractériser par leur structure chimique : c'est ainsi que l'on a appelé lipides les substances qui contiennent dans leur molécule des acides gras (mettant ainsi en évidence le rôle important et original de ces éléments) ; il s'agit le plus souvent d'esters, mais on rencontre aussi des amides d'acides gras (sphingolipides). On range à côté des lipides, des substances qui en sont proches par leur caractère de solubilité dans les solvants organiques (le cholestérol par exemple). 
Le cerveau, qualitativement, possède tous les lipides que I'on retrouve dans d'autres organes, et même dans le règne végétal, mais son originalité est d'en contenir certains en très grandes quantités (les sphingolipides par exemple), alors qu'ils sont mineurs dans d'autres organes.

Selon leur degré de complexité, les lipides sont classés, pour tout tissu, en trois catégories (tabl. 1).

\section{TABLEAU 1}

Nomenclature des principaux lipides

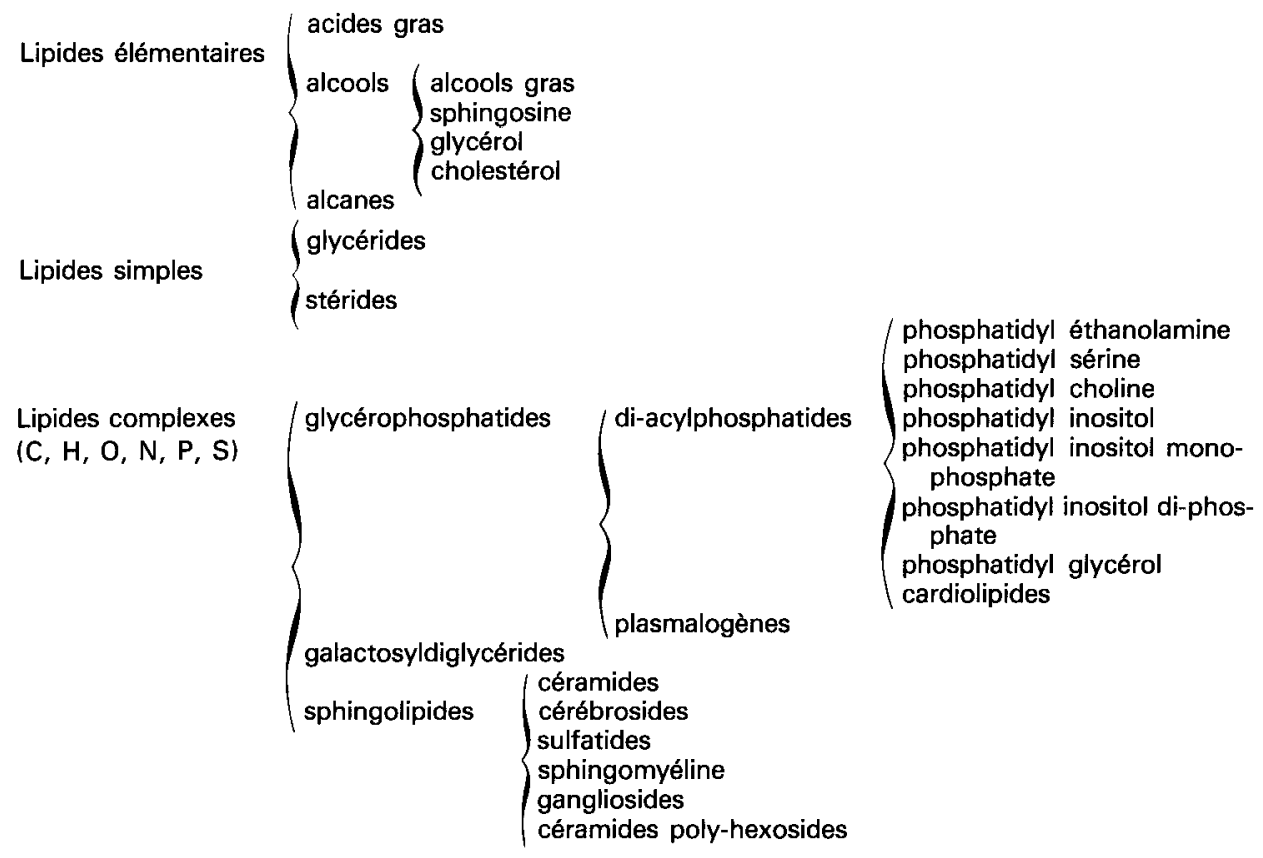

- les lipides élémentaires : parmi ceux-ci les acides gras et les alcools n'existent qu'à l'état de trace sous forme libre dans le tissu nerveux; par contre, ils interviennent dans les structures des lipides simples et complexes. Les alcanes sont présents en quantité relativement importante dans la myéline. Le cholestérol est une molécule quantitativement très importante dans les membranes nerveuses;

- les lipides simples : le système nerveux ne contient pratiquement pas de glycérides ; certains esters du cholestérol (stérides) sont présents à l'état de trace, et transitoirement, au cours du développement cérébral normal. Par contre ils n'apparaîssent en grande quantité qu'au cours des phénomènes de dégénérescence (normalement ils sont à peu près absents) ;

- les lipides complexes : la myéline, comme toute membrane, est riche en phospholipides. II faut noter la présence de plasmalogènes (liaison vinyl éther en 1 
Les cérébrosides sont synthétisés à partir des céramides en présence d'UDPgalactose ; la sulfatation des cérébrosides par la PAPS (3'-phosphoadénosine 5'phosphosulfate) qui donne le sulfate, produit les sulfatides. Inversement, la dégradation des sulfatides par une enzyme lysosomiale (une sulfatase) produit des cérébrosides; cette enzyme est mesurée in vitro sous forme d'aryl-sulfatase. Dans la leucodystrophie métachromatique, cette enzyme est déficiente, ce qui provoque une accumulation des sulfatides. Les galacto-cérébrosides sont à leur tour dégradés par un autre enzyme lysosomial, la galactosylcérébroside- $\beta$ galactosidase. Dans la maladie de Krabbe (leucodystrophie à cellules globoïdes), cette enzyme est déficiente. Enfin, un autre enzyme lyosomial, la céramidase dégrade les céramides; dans la maladie de Farber, elle est très diminuée.

Les sphingomyélines sont synthétisées principalement à partir des céramides en présence de CDP-choline ; leur dégradation se fait dans le lysosome grâce à une sphingomyélinase. Le défaut génétique de cet enzyme engendre une accumulation de sphingomyéline dans la maladie de Niemann-Pick (tabl. 2).

Les gangliosides sont synthétisés par addition séquentielle de monosaccharides ou d'acide $\mathrm{N}$-acetyl-neuraminique, catalysée par des glycosyltransférases spécifiques, généralement microsomiales. Les dégradations se font dans les lysosomes par des glycosidases et des neuraminidases; si l'une est perturbée, une lipidose apparaît (tabl. 2). L'activité enzymatique peut être mesurée à l'aide d'un analogue de structure, éventuellement sur une culture obtenue à partir d'un prélèvement amniotique.

Le système nerveux présente donc une grande richesse en lipides, qui le distingue nettement de tous les autres tissus de mammifères: les lipides constituent plus de la moitié du poids sec du cerveau et il s'agit essentiellement de lipides structuraux non énergétiques. Les acides gras constitutifs de ces lipides jouent un rôle particulier dans les propriétés des membranes cérébrales : la myéline contient de fortes quantités d'acides gras à très longues chaînes (Norton, 1977) puisqu'un acide gras sur six a un nombre d'atomes de carbone supérieur à dix-huit (alors que dans la substance grise, pauvre en myéline, il n'y en a qu'un sur cent). Par ailleurs les terminaisons nerveuses (synaptosomes) sont particulièrement riches en acides gras polyinsaturés. La présence de ces acides doit être mise en parallèle avec les propriétés biologiques et physicochimiques des membranes : par exemple les acides gras à très longues chaînes saturées et monoinsaturées sont les "chevilles " qui maintiennent l'édifice myélinique en place. II est donc particulièrement utile de connaître les origines des acides gras cérébraux.

Le cerveau contient deux types d'acides gras :

- acides gras saturés et monoinsaturés : à côté des longueurs de chaînes communes à tout organe (16 et 18 atomes de carbone, acide palmitique $C_{16: 0}$, stéarique $C_{18: 0}$, oléique $C_{18: 1}$ ) il y a de très longues chaînes, à $20,22,24$ voire 26 et 28 atomes de carbone. Ces dernières sont essentiellement trouvées dans les sphingolipides myéliniques. Les acides gras $\alpha$-hydroxylés sont directement dérivés des acides à très longues chaînes; ils sont les mêmes localisations subcellulaires :

- acides gras polyinsaturés, principalement à 20 et 22 atomes de carbone avec respectivement, 4 et 6 doubles liaisons. Ces acides sont dérivés d'acides essen- 
tiels, provenant donc exclusivement de la nutrition, car ne pouvant être élaborés par les mammifères: les acides linoléique $\left(C_{18: 2}\right)$ et linoléique $\left(C_{18: 3}\right)$. La synthèse des acides gras polyinsaturés n'a été que peu abordée dans le cerveau.

L'origine des acides gras cérébraux non essentiels doit donc être envisagée à deux niveaux :

A) Synthèse in situ : quel est le potentiel de synthèse du cerveau au cours de son développement; quels sont les mécanismes enzymatiques qui interviennent dans la synthèse des acides gras ; quelles sont leurs localisations et leur régulation?

B) Origine extra-cérébrale : y a-t-il un transport à travers la barrière hématoencéphalique ? Varie-t-il au cours du développement ? Quels sont les effets de certains types de malnutrition sur la composition en acides gras des structures cérébrales ? Pour répondre à ce dernier point, une hypotrophie in utero provoquée par clampage d'une artère utérine à été choisie.

\section{Synthèse des acides gras}

\section{A. - Synthèse des acides gras saturés et monoinsaturés.}

La palmitate synthétase, complexe multienzymatique cytosolique soluble, connue de la bactérie au mammifère, synthétise uniquement des chaînes moyennes $\left(C_{16}\right.$ : acide palmitique, principalement). Par contre, le lieu et le mode de synthèse des très longues chaînes ont dû être entièrement analysés, car ils étaient totalement inconnus quel que soit l'organe ou l'organisme; seule la localisation subcellulaire de leur synthèse était précisée (Aeberhard et Menkes, 1967). Toutefois l'injection de précurseurs radioactifs avait permis de proposer l'existence d'un mécanisme d'allongement, sans préciser sa localisation ni ses mécanismes (Murad et Kishimoto, 1978).

1) Synthèse microsomiale (Bourre et al., $1978 \mathrm{c}$ ). - Les microsomes contiennent plusieurs systèmes enzymatiques. Le premier est un système de novo élaborant de l'acide palmitique principalement. Le deuxième est un système d'allongement qui fait suite au complexe de novo pour former de l'acide stéatique. Le troisième reprend cet acide pour synthétiser des chaînes très longues ; seul ce dernier système est diminué de $60 \mathrm{p}$. 100 chez le mutant quaking et virtuellement absent chez le mutant jimpy, qui sont des mutants neurologiques présentant une myélinisation perturbée. Les acides gras monoinsaturés, très importants aussi au niveau de la myéline, sont synthétisés par le même complexe d'allongement que leurs homologues saturés (Bourre et al., 1976) mais à partir de l'oléyl-CoA, expliquant ainsi pourquoi saturés et monoinsaturés sont diminués chez le mutant quaking et justifiant la position en w9 de la double liaison à partir du méthyl terminal. Cet oléyl-CoA est lui-même obtenu à partir du stéaryl-CoA (Carreau et al., 1979) : la stéaryl-CoA désaturase est très active dans le cerveau (de même que d'ailleurs la palmityl-CoA désaturase).

2) Synthèse mitochondriale (Boone et Wakil, 1970 ; Paturneau-Jouas et al., 1976. - Les mitochondries cérébrales sont équipées pour la synthèse des acides 
gras. Les complexes enzymatiques sont intra-mitochondriaux car il faut rompre les organelles (sonication, détergent) pour révéler l'activité. Comme la fraction soluble et les microsomes, les mitochondries possèdent un système de novo. Elles ont aussi la possibilité d'allonger les acides gras, mais il n'y a qu'un seul complexe d'allongement après le système de novo (contrairement à ce qui se passe dans les microsomes). De plus, les mitochondries et les microsomes synthétisent les mêmes acides mais par des voies biochimiques totalement distinctes (substrats et cofacteurs différents). Les synthèses mitochondriales sont tout à fait normales chez les mutants quaking et jimpy : les mitochondries n'aident ni ne remplacent les microsomes défaillants. Elles ne sont pas liées aux phénomènes de myélinisation. Des comparaisons entre pouvoir de synthèse in vitro, vitesse de renouvellement in vitro, et analyse des lipides et acides gras mitochondriaux suggèrent que ces organelles sont autonomes.

3) Evolution ontogénétique des systèmes de biosynthèse des acides gras (Bourre et al., $1978 \mathrm{c}$ ). - L'activité du système de novo soluble (Cantrill et Carey, 1975 ; Volpe et al., 1973) (la palmitate synthétase) précède la myélinisation : elle est contemporaine de la multiplication cellulaire (elle joue donc un rôle de l'élaboration des membranes gliales). Les trois systèmes microsomiaux ont une évolution cohérente. Le premier (de novo) est maximal à $10-12$ jours ; la $\mathrm{C}_{16}$-élongase (qui synthétise de l'acide stéarique à partir de palmityl-CoA, lui-même produit de réaction du système précédent) a son acmé à 15 jours ; la $\mathrm{C}_{18}$-élongase à 18 jours.

En effet, il est normal que ces trois systèmes ne soient pas maxima simultanément à 18 jours (date de l'acmé de myélinisation et de dépôt des très longues chaînes) car en fait le dépôt de la myéline et sa maturation biochimique se font en deux temps : la membrane contient d'abord des sphingolipides formés d'acides gras à chaînes moyennes $\left(C_{16}\right.$ et $\left.C_{18}\right)$ puis, plus tard, constitués de très longues chaînes.

Ainsi, la synthèse de l'acide lignocérique $\left(\mathrm{C}_{24}\right)$ myélinique se fait dans les microsomes, le maximum du pouvoir synthétique observé in vitro (par mesure d'activités enzymatiques) correspondant à la vitesse maximale de dépôt (observée in vivo par calcul des quantités d'acides lignocérique trouvé dans le cerveau total ou la myéline au cours du développement cérébral). Ce maximum n'apparaît pratiquement pas chez le mutant quaking ; la synthèse est virtuellement absente chez les jimpy amyélinisés. II faut noter que l'efficacité synthétique du cerveau quaking est presque normale à 30 jours, mais le temps de myélinisation étant passé, il n'y a pas de récupération possible.

Le système d'allongement mitochondrial est normal chez les deux mutants au cours de tout leur développement cérébral. II croît faiblement mais régulièrement au cours de la maturation cérébrale. Le défaut microsomal n'est pas corrigé par une « hyperactivité compensatrice » mitochondriale.

4) Régulation des chaînes de synthèse dans les microsomes (Bernert et al., 1979). - Les deux systèmes d'allongement ne sont pas chacun le résultat d'une seule réaction enzymatique, mais la somme de plusieurs étapes intermédiaires dont les plus importantes sont la condensation ( $\alpha$-ceto acyl synthétase, qui condense la malonyl-CoA sur un acyl-CoA), la première réduction $(\alpha$ 
hydroxyacyl déshydrogénase) la déshydratation (acyl-déshydrogénase) et la deuxième réduction (enoyl-reductase). De plus des enzymes de transfert sont certainement présentes. Seule la condensation du malonyl-CoA sur le palmityl-CoA ou le stearyl-CoA sont différentes : ainsi l'individualité des deux élongases microsomales se situe au niveau de cette enzyme. De plus les réactions intermédiaires se font à des vitesses beaucoup plus grandes que la condensation qui est donc également l'étape limitante de la chaîne de synthèse (ceci explique que la vitesse de la condensation et la vitesse globale de toute la chaîne sont du même ordre de grandeur).

\section{B. - Synthèse des acides gras polyinsaturés.}

La synthèse des acides gras polyinsaturés n'a été que peu abordée dans le cerveau. Il est toutefois possible de présumer que les mécanismes y sont voisins sinon similaires à ceux trouvés dans d'autres organes, le foie en particulier. Ainsi, dans ce dernier organe, il a été montré que la désaturation requiert la présence d'ATP, de CoA, $\mathrm{Mg}^{++}, \mathrm{NADPH}$ ou NADH et $\mathrm{d}^{\prime} \mathrm{O}_{2}$. La double liaison est introduite au-delà des doubles liaisons existantes, en direction du groupement carboxyle : cela explique que les deux séries w6 et w3 soient inconvertibles sur le plan des voies de synthèse.

II existe plusieurs désaturases, chacune introduisant une double liaison dans une position donnée : $\Delta 6,5$ ou 4. II n'y a pas de $\Delta$ 8-désaturase, interdisant ainsi la formation de $C_{20}$ : 3w6 à partir de $C_{20}: 2 w 6$ et de $C_{20}: 4 w_{3}$ à partir de $C_{20}: 3 w 3$.

Les compétitions et les étapes limitantes de ces conversions ont été étudiées, et il faut retenir que les étapes d'élongation ne joueraient pas un rôle important dans la régulation des conversions.

Au sein d'une même série polyinsaturée, les métabolites plus longs et plus désaturés ont un effet inhibiteur sur la désaturation de leur précurseur : cela a été démontré in vitro pour la série w6 et la série w3 : I'inhibition de la conversion du $C_{18: 2 w 6}$ en $C_{18: 3 w 6}$ par l'acide arachidonique $\left(C_{20}: 4 w 6\right)$ en est un exemple. Ces systèmes sont assimilables à des mécanismes de contrôle rétroactif. Les mêmes désaturases étant utilisées par les deux séries w6 et w3, on comprend qu'il existe des phénomènes d'inhibition compétitive entre elles.

Dans le cerveau, l'anabolisme commence avec les acides gras essentiels $\left(C_{18: 2 w 6}\right.$ et $\left.C_{18: 3 w_{3}}\right)$ qui, provenant de l'alimentation, peuvent être captés par le cerveau, avant ou après élongation et désaturation. Ces deux mécanismes ayant lieu dans les microsomes, ces acides gras sont métabolisés sous la forme du dérivé acyl-CoA (Cook, 1978). Le processus d'élongation des acides gras polyinsaturés est supposé semblable à celui des chaînes saturées, les unités à 2 carbones étant apportées par la malonyl-CoA : il n'y aurait pas de système enzymatique spécifique à chaque série. Toutefois il faut noter que les cinétiques de condensation du malonyl-CoA sur les linoléyl-CoA et linolényl-CoA ne sont pas similaires : il n'est pas exclu que les allongements intervenant dans les séries w3 et w6 soient différents (Bourre et al., données non publiées). De plus l'allongement des acides gras polyinsaturés est moins atteint que l'allongement de leurs homologues saturés dans certains cas pathologiques (Bourre et al., 1976). 


\section{Origine extra-cérébrale des acides gras}

Les résultats enzymatiques obtenus in vitro comparés aux vitesses de renouvellement in vivo et à la quantité d'acides gras déposés au cours du développement, nous ont montré que le cerveau ne peut élaborer que $60 \mathrm{p} .100$ environ de ses acides gras. Une seule alternative peut donc être évoquée : une origine plasmatique, voire nutritionnelle. Les origines éventuelles ont été analysées en utilisant deux méthodes : l'une consiste à suivre la destinée d'un acide gras marqueur dans le sang, puis dans les structures cérébrales. L'autre met à profit l'existence d'hypotrophie pouvant être induite par ligature d'une artère utérine chez le rat (Minkowski et al., 1974).

\section{A. - Transport d'acides gras non essentiels traceurs à travers la barrière hémato-encéphalique.}

L'acide palmitique radioactif, injecté ou donné dans la nourriture, peut être capté par le cerveau (Dhopeshwarkar, 1975) : I'acide stéarique radioactif, injecté par voie veineuse, sous-cutanée ou intra-ventriculaire (injection stéréotaxique) est retrouvé dans le cerveau. Ceci est bien visualisé par radio-autographie suivie de colorations histologiques (Bourre et al., 1979). Par ailleurs des préparations cellulaires et subcellulaires montrent que l'acide stéarique radioactif injecté par voie sous-cutanée passe dans le cerveau où il est intégré au niveau des structures cérébrales (Bourre et al., 1979), la myéline tout particulièrement, mais aussi les synaptosomes. De plus cet acide est nécessaire à l'élaboration des membranes de neurones et des astrocytes (Morand et al., 1979). Les phospholipides sont les principaux accepteurs, mais les spingolipides utilisent aussi l'acide gras traceur. L'acide stérique injecté est incorporé directement, tel quel, dans les membranes, ou bien il est métabolisé in situ avant la fixation au niveau des lipides : allongement en chaînes plus longues formant les acides arachidique, béhénique et lignocérique ou synthèse de chaînes plus courtes comme l'acide palmatique à partir d'éléments du catabolisme.

La relation sang-cerveau pour l'acide stéarique varie au cours du développement cérébral. En effet la captation totale exprimée en radioactivité par lipides d'un cerveau, est maximale vers 18 jours (période d'active myélinisation). Par contre l'activité " spécifique » calculée en radioactivité/mg de lipides est optimale les jours suivants la naissance des animaux (époque de multiplication cellulaire). Ces résultats montrent que pendant la myélinisation l'apport exogène est quantitativement important ; mais la synthèse in situ étant elle-même considérable, il y a dilution de la participation exogène, expliquant la faible activité spécifique. Par contre la contribution exogène est quantitativement plus faible pendant la multiplication gliale (car il y a moins de besoins), mais son rôle est essentiel, car la synthèse in situ est relativement réduite (d'où une forte activité spécifique).

Avec quelques variations selon l'âge considéré, l'acide stéarique est partiellement métabolisé dans le cerveau, soit par allongement (direct ou précédé d'une désaturation en acide oléique) soit par dégradation (avec production d'unités acé- 
tate qui seront réutilisées éventuellement pour élaborer de nouvelles molécules d'acides gras ou de cholestérol. Les événements ne sont pas exactement synchrones à l'échelle moléculaire : si chaque lipide est considéré individuellement, le maximum d'incorporation de l'acide stéarique se situe à 15 jours pour la phosphatidyl-choline et les cérébrosides, 18 jours pour la phosphatidyl éthanolamine, 21 pour la phosphatidyl-sérine (pour les radioactivités totales par cerveau).

Ainsi l'apport exogène en acides gras non essentiels est important pendant les deux périodes cruciales du développement postnatal (de la souris) : la multiplication gliale et la myélinisation. La synthèse in situ est elle-même active à ces époques. II y a un équilibre entre synthèse in situ et apport exogène.

\section{B. - Transport d'acides gras essentiels.}

La position $\beta$ des phospholipides est généralement occupée par un acide gras polyinsaturé, qui est le plus souvent le $20: 4$ w6, le $22: 5$ w3 et le $22: 6$ w3. Ces acides proviennent tels quels du sang ou bien sont dérivés des acides essentiels linoléique et linolénique après allongement et désuturation. Une carence en acides essentiels provoquera des anomalies au cours du développement cérébral, période où le besoin est particulièrement important (Crawford et Sinclair, 1972). Le tissu nerveux compensera partiellement ce déficit en synthétisant des acides polyinsaturés en w9 qu'il incorporera dans toutes ses membranes (Paoletti et Galli, 1972). Si un seul des acides essentiels est absent de la nourriture, le cerveau maintiendra constante sa teneur en acides polyinsaturés, mais il y aura un déficit en très longues chaînes qui auraient dû dériver de cet acide absent ; de plus, il existe une concentration minimale d'acide essentiel dans la nourriture, au-dessous de laquelle le cerveau ne peut plus maintenir sa composition sinon au détriment d'autres organes (Nouvelot et al., 1981).

\section{C. - Effets de l'hypotrophie intra-utérine Imodèle CIUR: croissance intra- utérine retardée).}

Ce modèle est obtenu en pratiquant la ligature d'une artère utérine chez une rate gravide : cette opération est effectuée au $17^{\bullet}$ jour de la gestation (Wiggelsworth, 1964). La réduction du flux sanguin vers le fœetus est le premier mécanisme impliqué dans le modèle CIUR. Certaines lésions vasculaires équivalentes ont d'ailleurs été décrites chez le nouveau-né "small for date " (Minkowski et al., 1974). L'apport sanguin fœtal ramené au poids corporel est réduit ; cependant, on sait qu'il y a un flux sanguin préférentiel vers le cerveau fotal, comparativement au foie. Le cerveau serait donc plus particulièrement protégé contre les carences nutritionnelles.

En réalité, I'hypotrophie touche également le cerveau : la réduction du poids cérébral du jeune rat hypotrophique en est la première manifestation (le poids du cerveau du rat hypotrophique adulte est subnormal). Cela ne s'accompagne pas d'une variation sensible de la taille des cellules, mais est accompagné d'une diminution du nombre de cellules nerveuses. Cette diminution peut être due au ralentissement de la multiplication cellulaire.

Ce type de malnutrition prénatale a retenu notre attention, car il concerne 
une période bien particulière du développement cérébral. Les neurones sont déjà formés à partir des neuroblastes initiaux ; les cellules gliales ne sont pas encore en place et la myéline est absente. Entre le $17^{\mathrm{e}}$ jour de la gestation et la naissance, le doublement de poids du cerveau s'accompagne d'une accumulation considérable de matériaux simultanément à la croissance axonale et dendritique, et à l'établissement des connections synaptiques. Sachant que la chronologie du développement cérébral est très précisément programmée, un retard de maturation, lié au déficit permanent en nombre de cellules, pourrait être irréversible. Connaissant les décalages qui existaient dans le développement des différentes populations cellulaires cérébrales, nous supposons que seules les structures cellulaires ou subcellulaires se développant au moment de la malnutrition sont susceptibles d'être atteintes (les neurones par exemple).

Par ailleurs la composition en acides gras des cellules cérébrales est un repère intéressant car elle est à la fois un index de maturation du cerveau et un index de variations qualitatives de la membrane cellulaire (Morand et al., 1981) (cela a été aussi très bien observé dans le cas des carences en acides gras essentiels). Les deux indices peuvent être proposés :

- le rapport du taux d'acides gras saturés $\left(C_{16: 0}\right.$ à $\left.C_{24: 0}\right)$ sur le taux de monoinsaturés $\left(\mathrm{C}_{16: 1}\right.$ à $\left.\mathrm{C}_{24: 1}\right)$;

- le rapport série w6 sur série w3.

Les neurones des animaux hypotrophiques présentent essentiellement deux modifications : un déficit en acides gras monoinsaturés, compensé par une élévation des taux d'acides gras saturés, et un déséquilibre important entre les séries polyinsaturées w6 et $w 3$, au profit de la série w3 (le total w6 + w3 reste inchangé comparativement au groupe témoin). Les oligodendrocytes présentent ce même déficit en acides gras monoinsaturés et de plus nous retrouvons aussi un déséquilibre entre les séries w6 et w3, mais au profit de la série w6.

La phase de mise en place des neurones étant prénatale, l'altération de leur membrane paraît assez logique. Par contre, les oligodendrocytes se développant après la naissance, les modifications de la composition en acides gras de ce type cellulaire ne sont pas actuellement explicables. La quantité d'acides gras polyinsaturés (w6 + w3) semble être maintenue et contrôlée par le tissu nerveux alors que la répartition entre les deux séries est beaucoup plus sensible aux facteurs exogènes (Morand et al., 1981).

\section{Conclusion.}

A. - Le système nerveux est extrêmement riche en lipides, donc en acides gras. Ces derniers constituent le squelette de toute membrane biologique; les membranes cérébrales sont formées d'acides gras de nature particulière et pouvant être mis en parallèle avec leurs fonctions biologiques. Ainsi la myéline est très riche en sphingolipides lauxquels est associée toute une pathologie chez l'homme) ; ces sphingolipides sont formés d'acides gras à très longues chaînes saturées et monoinsaturés, $\alpha$-hydroxylés éventuellement. Les terminaisons nerveuses sont riches en acides gras polyinsaturés, qui dérivent d'acides gras essentiels. 
B. - Il y a une relation précurseur-produit entre la myéline et les microsomes. Les acides gras myéliniques sont synthétisés au niveau de ces derniers. La biosynthèse des acides gras dans les microsomes de cerveau procède en trois étapes par l'intermédiaire de trois complexes enzymatiques distincts. Seul le troisième (allongeant des acides gras de 18 atomes de carbone) est diminué chez quaking et absent chez jimpy (mutants dysméliniques) et sans doute aussi absent dans un cas de leucodystrophie humaine (Bourre et al., 1978 a). De plus, la synthèse des acides saturés et monoinsaturés est faite par le même complexe d'allongement à partir de l'oleyl-CoA. A l'acmé de la myélinisation, la synthèse est maximale (elle est beaucoup plus faible avant et réduite après). La différence entre les deux systèmes d'allongement ainsi que la régulation de toutes les chaînes de synthèse se font au niveau de la première enzyme, qui condense le malonyl-CoA (donneur de carbone) sur l'acyl-CoA.

Ontogénétiquement, le $2^{\circ}$ système d'allongement apparaît en même temps que les autres systèmes de synthèse des lipides myéliniques : les inductions des enzymes impliqués dans la synthèse des lipides myéliniques se font simultanément et non de manière séquentielle (Tennekoon et al., 1980).

Les mitochondries sont aussi capables de synthétiser les acides gras à très longues chaînes, mais par des mécanismes biochimiques totalement différents des mécanismes microsomiaux. Cette synthèse n'est pas en rapport avec la myélinisation; de plus, elle est normale chez des mutants dysméliniques : ceci est en faveur d'une autonomie des mitochondries, même dans le cerveau. Le complexe soluble synthétise des chaînes courtes et moyennes. II est en rapport avec la multiplication cellulaire (période pré-myélinique). La régulation génétique des systèmes n'est pas la même dans le rein et le cerveau (Bourre et al., 1975). Le système nerveux périphérique synthétise les acides gras selon un schéma tout à fait analogue au système nerveux central.

Les acides gras synthétisés dans le réticulum (Cassagne et al., 1978) peuvent être métabolisés en alcanes (Cassagne et al., 1977) et en alcools gras (Bourre et Daudu, $1978 \mathrm{~b}$ ). Les premiers seront à leur tour directement incorporés dans la myéline (Bourre et al., 1977). Les seconds seront utilisés pour synthétiser des molécules essentiellement myéliniques, les plasmalogènes.

C. - Le cerveau ne synthétise pas tous les acides gras nécessaires à l'élaboration de ses membranes : l'origine exogène (nutritionnelle ?) a été démontrée en injectant par voie veineuse et sous-cutanée des acides gras radioactifs. Ceux-ci sont captés par le cerveau qui les utilise pour construire ses membranes (soit en les intégrant directement tels quels, soit après les avoir allongés ou désaturés). De plus, la relation sang-cerveau varie au cours du développement cérébral : la captation d'acide sérique par le cerveau est quantitativement importante pendant la myélinisation, qualitativement essentielle pendant la multiplication cellulaire.

Les acides gras non essentiels sont cependant indispensables au cours du développement cérébral : une hypotrophie intrautérine provoque de graves anomalies dans la composition en acides gras des neurones et oligo-dendrocytes accompagnées d'une réduction quantitative de la myéline.

7e Réunion du groupe Développement I.N.R.A., Nouzilly/Tours, 14-15 mai 1981. 


\section{Reférences}

AEBERHARD E., MENKES J., 1967. Biosynthesis of long chain fatty acids by subcellular particles of mature brain. J. biol. Chem., 243, 3834-3840.

BERNERT J. T., BOURRE J. M., BAUMANN N., SPRECHER H., 1979. Fatty acid biosynthesis in mouse brain microsomes : the activity of partial reactions involved in the chain elongation of palmitoyl-CoA and stearoyl-CoA. J. Neurochem., 32, 85-90.

BOONE S., WAKIL S., 1970. In vitro synthesis of lignoceric acid and nervonic acid in mammalian liver and brain. Biochemistry, 9, 1470-1479.

BOURRE J. M., DAUDU O., BAUMANN N., 1975. Fatty acid biosynthesis in mice brain and kidney microsomes, comparison between quaking mutant and control. J. Neurochem., 24, 1095-1097.

BOURRE J. M., DAUDU O., BAUMANN N., 1976. Nervonic acid biosynthesis by erucyl-CoA elongation in normal and quaking mouse brain microsomes. Elongation of other unsaturated fatty acyl-CoA (mono- and poly- unsaturated). Biochim. biophys. Acta, 424, 1-7.

BOURRE J. M., CASSAGNE C., LARROUQUĖRE-REGNIER é., DARRIET D., 1977. Occurrence of alkanes in brain myelin. Comparison between normal and quaking mouse. J. Neurochem., 29, 645-648.

BOURRE J. M., BORNHOFEN J. H., ARAOZ C., DAUDU O., BAUMANN N., 1978 a. PealizaeusMerzbacher disease : brain lipid and fatty acid composition. J. Neurochem., 30, 719-727.

BOURRE J. M., DAUDU O., 1978 b. Stearyl-alcohol biosynthesis from stearyl-CoA in mouse brain microsomes in normal and dysmyelinating mutants (quaking and jimpy). Neurosci. Letters, 7, 225-230.

BourRe J. M., POLLet S., PATURNEAU-JOUAS M., BAUMANN N., $1978 \mathrm{c}$. Fatty acid biosynthesis during brain development, 17-26. In S. GATT, L. FREYSZ, P. MANDEL, Enzymes of lipid metabolism, Plenum Publ. Co., New York.

BOURRE J. M., GOZLAN-DEVILLIERRE N., MORAND O., BAUMANN N., 1979. Importance of exogenous saturated fatty acids during brain development and myelination in mice. Ann. Biol. anim. Biochim. Biophys., 19, 173-180.

CANTRILL R., CAREY E., 1975. Changes in the activities of de novo fatty acid synthesis and palmityl-COA synthetase in relation to myelination rabbit brain. Biochim. biophys. Acta, 38, 165-175.

CARREAU J. P., DAUDU O., PAZLIAK P., BOURRE J. M., 1979. Palmityl-CoA and stearyl-CoA desaturase in mouse brain microsomes during development in normal and neurological mutants (quaking and jimpy). J. Neurochem., 32, 659-660.

CASSAGNE C., DARRIET D., BOURRE J. M., 1977. Evidence of alkane synthesis by the sciatic nerve of the rabbit. FEBS Letters, 82, 51-54.

CASSAGNE C., DARRIET D., BOURRE J. M., 1978. Biosynthesis of very long chain fatty acids by the sciatic nerve of the rabbit. FEBS Letters, 90, 336-340.

COOK H. W., 1978. In vitro formation of polyunsaturated fatty acids by desaturation in rat brain : some properties of the enzyme in developing brain and comparison with liver. $J$. Neurochem., 30, 1327-1334.

CRAWFORD M. A., SINCLAIR A. J., 1972. Nutritional influences in the evolution of mammalian brain, 267-292. In Lipid, malnutrition and the developing brain, Ciba Found. Symp., Elsevier, Ainsterdam.

DHOPESHWARKAR G., 1975. Uptake and transport of fatty acids into the brain and the role of the blood brain barrier system. Adv. Lipid Biochem., 11, 104-142.

MINKOWSKI A., ROUX J., TORDET-CARIDROIT C., 1974. Pathophysiologic changes in intrauterine malnutrition, 45-78. In KUSICK, Nutrition and fetal development, Karger, Bale.

MORAND O., BAUMANN N., BOURRE J. M., 1979. In vivo incorporation of exogenous ${ }^{1-14} \mathrm{C}$ stearic acid into neurons and astrocytes. Neurosci. Letters, 13, 177-181.

MORAND O., CHANEZ E., MASSON M., DUMONT O., FLEXOR M., BAUMANN N., BOURRE J. M., 1981. Modification of brain cells fatty acid composition induced by intrauterine under-nutrition. J. Neurochem., 37, 1057-1060. 
MURAD S., KISHIMOTO Y., 1978. Chain elongation of fatty acid in brain : a comparison of mitochondrial and microsomal enzyme activities. Arch. Biochem. Biophys., 30, 300-306.

NORTON W. T., 1977. Chemical pathology of disease involving myelin, 383-408. In MORELL P., Myelin, Plenum Press, New York.

NOUVELOT A., BOURRE J. M., JEZILLE G., DEWAILLY P., JAILLARD J., 1981. Polyunsaturated fat acid alteration in brain phospholipids during development in rats fed with peanut and rapeseed oil taking into account differences between milk and maternal food. (Soumis à $\mathrm{Am}$. Nutr. Metab.).

PAOLETTI R., GALLI G., 1972. Effects of essential fatty acid deficiency on the central nervous system in the growing rat, 121-140. In Lipid, malnutrition and the developing brain. Ciba, Found. Symp., Elsevier, Amsterdam.

PATURNEAU-JOUAS M., BAUMANN N., BOURRE J. M., 1976. Elongation of palmityl-CoA in mouse brain mitochondria: comparison with stearyl-CoA. Biochem. biophys. Res. Commun., 71, 1326-1334.

SUZUKI K., 1976. Chemistry and metabolism of brain lipids, 308-327. In SIEGEL T., ALBERTS R., KATZMAN R., AGRANOFF B. Basic neurochemistry, Little Brown and Co., Boston.

TENNEKOON G. I., KISHIMOTO Y., SINGH I., NONAKA G., BOURRE J. M., 1980 . The differentiation of oligodendrocytes in the rat optic nerve. Dev. Biol., 79, 149-158.

VOLPE J., LYLES T., RONCAR D., VAGELOS P., 1973. Fatty acid synthetase of developing brain and liver. J. biol. Chem., 248, 2502-2513.

WIGGELSWORTH J. S., 1964. Experimental growth retardation in the fetal rat. J. Path. Bact., 88, 1-33. 